\title{
CHARACTERIZATION OF A NEW ARYL ALKANONE AND OTHER COMPOUNDS PRESENT IN HORSFIELDIA IRYA SEEDS
}

\author{
S. WIMALASENA and E. KARUNAWANSHA \\ Department of Chemistry, University of Kelaniya, Kelaniya.
}

(Received: 15 April1994; accepted: 06 November 1994)

\begin{abstract}
A new aryl alkanone 1-(2,6-dihydroxyphenyl)dodecan-1-one, trimyristin, myristic acid, asarinin and horsfieldin were isolated from the methanolic extract of seeds of Horsfieldia irya. The petrol extract gave trimyristin and myristic acid. The isolated compounds were characterized using spectral data.
\end{abstract}

Key words: Horsfieldia irya, Myristicaceae, horsfieldin, asarinin, 1-(2,6 dihydroxyphenyl)dodecan-1-one, myristic acid, trimyristin.

\section{INTPODUCTION}

The family Myristicaceae comprises about 380 species $^{1}$ but only six members of this family are found in Sri Lanka. ${ }^{2}$ They are Myristica fragrans, Myristica dactyloides, Myristica zeylanica, Horsfieldia irya, Horsfieldia iryaghedhi, Myristica salicifolia ${ }^{2}$ Horsfieldia irya (S- Iriya) is a large tree found in Sri Lanka, Burma, Andaman Islands, Java and Malacca islands. ${ }^{3}$ It has a dark purple grey bark and the branches are found at the top of the tree. The fruit is rust coloured, and about $2.5 \mathrm{~cm}$ in diameter. It has a leathery rusty tomentose outside pericarp. It dehiseces into two halves and the seed is covered entirely by a scarlet aril. Horsfieldia irya is used in traditional medicine. The latex from it is used to clean ulcers and the root macerated with lime juice is drunk as a remedy for snake bite poisoning. The leaves are used to draw pus from boils and sores. ${ }^{3}$ No study has been previously reported on this plant and this paper reports the isolation of trimyristin $(0.62 \%)$, myristic acid $(0.34 \%)$, 1-(2,6-dihydroxyphenyl)dodecan-1one $(0.12 \%)$, asarinin $(0.11 \%)$, and horsfieldin $(0.09 \%)$ from the seeds of this plant.1-(2,6-dihydroxyphenyl)dodecan-1-one is a new compound. Structurally related arylalkanones have been previously isolated from Myristica dactyloides ${ }^{4,5}$ and M.malabarica. ${ }^{6}$ Horsfieldia iryaghedhi gave a related compound dodecanoyl phloroglucinol. ${ }^{7,8}$ The isolation of lignans asarinin and horsfieldin from Horsfieldia iryaghedhi ${ }^{7}$ and trimyristin and myristic acid from Horsfieldia iryaghedhi $i^{7}$ and Myristica fragrans ${ }^{9}$ have been previously reported. Unlike Myristica fragrans which is a rich source of essential oils Horsfieldia irya did not yield any volatile oil on steam distillation.

\section{METHODS AND MATERIALS}

Melting points were uncorrected and determined using electrothermal melting point apparatus. Identity of the compounds was established by mixed melting point IR, ${ }^{1} \mathrm{H}$ NMR, ${ }^{13} \mathrm{C}$ NMR and MS. Petrol refers to the fraction of bp $40-60^{\circ} \mathrm{C}$. Vacuum liquid chromatography and TLC were carried out on silica gel using petrol/ethyl acetate mixtures. ${ }^{1} \mathrm{H}$ NMR spectra, ${ }^{13} \mathrm{C} \mathrm{NMR}$ were recorded in $\mathrm{CDCl}_{3}$ with TMS as internal standard. MS and NMR spectra were recorded the 
Departments of Chemistry of the University of Glasgow, University of Otago and University of Arizona. UV spectra were recorded in EtOH on Shimadzu UV 160.

Extraction and isolation: Dried powdered seeds of Horsfieldia irya $(100 \mathrm{~g})$ obtained from Matale were extracted with hot petrol and hot methanol.Petrol extract gave a white solid (38.93g). This solid ( $10 \mathrm{~g})$ was subjected to vacuum liquid chromatography on silica gel. Recrystallization with methanol of the fractions eluted with petrol and petrol:ethyl acetate 99:1 gave a white solid trimyristin $(410 \mathrm{mg})$ and white needles of myristic acid $(230 \mathrm{mg})$ respectively. The reddish brown gum ( $40 \mathrm{~g}$ ) was obtained from the methanolic extract. This gum $(10 \mathrm{~g})$ was subjected to vacuum liquid chromatography. The fraction eluted with petrol gave trimyristin $(210 \mathrm{mg})$ and we fraction eluted with petrol :ethyl acetate 99:1 gave myristic acid(110mg) which were identified by mixed $\mathrm{mp}$ and IR, ${ }^{1} \mathrm{H}$ NMR. Recrystallization of the fractions eluted with a mixture of petrol: ethyl acetate $19: 1,9: 1,7: 3$ gave pale yellow needles of 1-(2,6- dihydroxyphenyl) dodecan1 -one $(120 \mathrm{mg})$, colourless needles of asarinin $(110 \mathrm{mg})$ and horsfieldin $(90 \mathrm{mg})$ respectively.

\section{RESULTS AND DISCUSSION}

Column chromatography of the petrol extract of the dried seeds of Horsfieldia irya yielded trimyristin and myristic acid which were identified by comparison of ${ }^{1} \mathrm{H}$ NMR, ${ }^{13} \mathrm{C}$ NMR and $\mathrm{mp}$ of the authentic samples.

\section{Trimyristin:}

$\mathrm{mp} 54-56^{\circ} \mathrm{C}\left(\mathrm{Lit}^{7} 54-55^{\circ} \mathrm{C}\right)$;

$\operatorname{IR}\left(v_{\max }\right)\left(\mathrm{CHCl}_{3}\right) 2928,2855,1734,1635,1214$.

${ }^{1} \mathrm{H}$ NMR (300MHz, $\mathrm{CDCl}_{3}$ ) ( $\left.\delta \mathrm{ppm}\right) 5.30-5.20, \mathrm{~m}, 1 \mathrm{H}, \mathrm{CH}-\mathrm{O} ; 4.33-4.11, \mathrm{~m}, 4 \mathrm{H}$, $\mathrm{CH}_{2} \mathrm{O} ; 2.32, \mathrm{t}, 6 \mathrm{H}, \mathrm{J}=7.5 \mathrm{~Hz}, \mathrm{COCH}_{2} ; 1.65-1.55, \mathrm{~m}, 6 \mathrm{H}, \mathrm{CO}-\mathrm{CH}_{2}-\mathrm{CH}_{2}: 1.35-1.17, \mathrm{~m}$, $6 \mathrm{OH}, \mathrm{CH}_{2} ; 0.88, \mathrm{t}, 9 \mathrm{H}, \mathrm{J}=6.8 \mathrm{~Hz}, \mathrm{CH}_{3}$.

${ }^{13} \mathrm{C} \mathrm{NMR}\left(50 \mathrm{MHz} \mathrm{CDCl}_{3}\right)(\delta \mathrm{ppm}) 173.27,172.85,68.84,62.08,34.20,34.03$, $31.91,29.65,29.48,29.35,29.27,29.11,24.87,24.85,22.68,14.10$.

\section{Myristic acid:}

mp 55-57 ${ }^{\circ} \mathrm{C}\left(\mathrm{Lit}^{10} 58^{\circ} \mathrm{C}\right)$ mixed $\operatorname{mp} 55-57^{\circ} \mathrm{C}$.

$\mathrm{IR}\left(v_{\max }\right)\left(\mathrm{CHCl}_{3}\right) 3500-3000,2926,2855,1709,1467,1213$.

${ }^{1} \mathrm{H}$ NMR $\left(300 \mathrm{MHz}_{2} \mathrm{CDCl}_{3}\right)(\delta \mathrm{ppm}) 8.25, \mathrm{~s}, 1 \mathrm{H}, \mathrm{COOH} ; 2.34, \mathrm{t}, 2 \mathrm{H}, \mathrm{J}=7.4 \mathrm{~Hz}$, $\mathrm{COCH}_{2} ; 1.68-1.58, \mathrm{~m}, 2 \mathrm{H}, \mathrm{COCH}_{2} \mathrm{CH}_{2} ; 1.2-1.4, \mathrm{~m}, 20 \mathrm{H}, \mathrm{CH}_{2} ; 0.88, \mathrm{t}, 3 \mathrm{H}, \mathrm{J}=7 \mathrm{~Hz}$, $\mathrm{CH}_{3}$.

${ }^{13} \mathrm{C} \mathrm{NMR}\left(75 \mathrm{MHz} \mathrm{CDCl}_{3}\right)(\delta \mathrm{ppm}) 180.43,34.17,32.00,29.72,29.67,29.51,29.44$, $29.32,29.14,24.74,22.77,14.20$.

In addition to these the methanolic extract gave 1-(2,6-dihydroxyphenyl) dodecan-1-one, asarinin and horsfieldin which were characterized as follows. 


\section{1-(2,6-Dihydroxyphenyl)dodecan-1-one:} mp $61-62^{\circ} \mathrm{C}$.

UV $\lambda_{\max }(\mathrm{nm}) 226,284, \lambda_{\max }^{\mathrm{EtOH} / \mathrm{OH}^{-}}$(nm) 236, 318;

IR ( $\left.v_{\text {mox }}\right)\left(\mathrm{CCl}_{)}\right) 3600,2920,1635,1610,1450,1245,1035,815,790,755$.

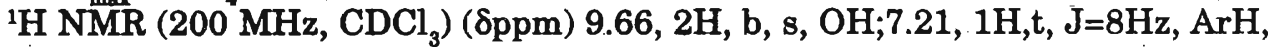
$6.38,2 \mathrm{H}, \mathrm{d}, \mathrm{J}=8 \mathrm{~Hz}, \mathrm{ArH} ; 3.12,2 \mathrm{H}, \mathrm{t}, \mathrm{J}=7.5 \mathrm{~Hz}, \mathrm{COCH}_{2} ; 1.69,2 \mathrm{H}, \mathrm{m}, \mathrm{COCH}_{2} \mathrm{CH}_{2}$; $1.25,16 \mathrm{H}, \mathrm{b}, \mathrm{s}, \mathrm{CH}_{2} ; 0.87,3 \mathrm{H}, \mathrm{t}, \mathrm{J}=6.6 \mathrm{~Hz}, \mathrm{CH}_{3}$;

${ }^{19} \mathrm{C} \mathrm{NMR}\left(50.5 \mathrm{MHz}^{2}, \mathrm{CDCl}_{\mathrm{g}}\right)(\mathrm{\delta pm}) 208.14,161.14,135.72,110.03,108.45,44.82$, $31.9,29.64,29.56,29.41,29.35,24.45,22.69,14.12$.

MS (m/z) (rel.int.\%) $292(2)\left[\mathrm{M}^{+}\right], 274$ (20), $246(5), 189(7), 165$ (25), $152(21), 147(75), 137(100), 123(15)$.

High resolution MS $\left[\mathrm{M}^{+}\right] 292.2052\left(\mathrm{C}_{18} \mathrm{H}_{28} \mathrm{O}_{3}\right.$ requires $\left.\left[\mathrm{M}^{+}\right] 292.2038\right)$.

The molecular peak at $\mathrm{m} / \mathrm{z}$ in the high resolution MS at 292.2052 indicated a molecular formula of $\mathrm{C}_{18} \mathrm{H}_{28} \mathrm{O}_{3}$. The IR spectrum showed the presence of a phenolic hydroxyl group $\left(v_{\max } 3600 \mathrm{~cm}^{-1}\right)$. The phenolic nature of the hydroxyl group was confirmed by the bathochromic shifts in the UV on addition of $\mathrm{NaOH}$. A hydrogen bonded carbonyl group was shown in the IR $\left[v_{\max } 1635 \mathrm{~cm}^{-1}\right] .{ }^{1} \mathrm{H} N M R$ showed a 1,2,3 trisubstituted $\mathrm{C}_{6} \mathrm{H}_{6}$ ring. The coupling constant $(8 \mathrm{~Hz})$ confirmed the presence of ortho coupled aromatic protons. $\mathrm{A}-\mathrm{CH}_{2} \mathrm{CH}_{2}$ group attached to a carbonyl group was indicated by the triplet at $\delta 3.12$ and extended polyethylene group by the multiplet at $\delta 1.25$. The distorted triplet at $\delta 0.87$ indicated the presence of $\mathrm{CH}_{3}$ group attached to a $\mathrm{CH}_{2}$. The base peak in the MS at $\mathrm{m} / \mathrm{z} 137$ arose by the cleavage $\alpha$ to the carbonyl group. The ${ }^{1} \mathrm{H} \mathrm{NMR}$ and ${ }^{13} \mathrm{C}$ NMR were similar to those previously reported for 1-(2,6-dihydroxyphenyl)tetradecan-1one. ${ }^{6}$ The above spectral data confirmed that the structure of the new compound is 1-(2,6- dihydroxyphenyl)dodecan-1-one.

\section{Asarinin:}

mp 120-122 ${ }^{\circ} \mathrm{C} .\left(\mathrm{Lit}^{7} 120-122^{\circ} \mathrm{C}\right) .(\alpha)_{\mathrm{D}}+117.8\left(\mathrm{Lit}^{7}(\alpha)_{\mathrm{D}}+120\right) \mathrm{IR} v_{\max }\left(\mathrm{CCl}_{4}\right) 2960$, $2880,1500,1490,1440,1300,1290,1050,970,790,780$.

${ }^{1} \mathrm{H} \mathrm{NMR}\left(200 \mathrm{MHz} \mathrm{CDCl}_{3}\right)(\mathrm{ppm}) 6.99-6.77, \mathrm{~m}, 6 \mathrm{H}, \mathrm{ArH} ; 5.95, \mathrm{~s}, 4 \mathrm{H}, \mathrm{OCH}_{2} ; 4.81$, $\mathrm{d}, \mathrm{J}=5 \mathrm{~Hz}, 1 \mathrm{H}, \mathrm{ArCHO} ; 4.38$, d, J=7Hz, $1 \mathrm{H}, \mathrm{ArCHO} ; 4.06, \mathrm{~d}, \mathrm{~J}=9 \mathrm{~Hz}, 1 \mathrm{H}, \mathrm{CH} ; 3.83-$ 3.77, $\mathrm{m}, 2 \mathrm{H}, \mathrm{CH}_{2} ; 3.31-3.27, \mathrm{~m}, 2 \mathrm{H}, \mathrm{CH}_{2} ; 2.95-2.85, \mathrm{~m}, 1 \mathrm{H}, \mathrm{CH}$.

Mass spectra $(\mathrm{m} / \mathrm{z})$ (rel. intensity\%) $354(33)[\mathrm{M}]^{+}, 203(14), 178(13), 161(25)$, 150(39), 149(100), 135(43) 131(23), 122(20), 121(19).

${ }^{13} \mathrm{C}$ NMR(50.5 $\mathrm{MHz} \mathrm{CDCl}_{3}$ ) (8ppm) 147.93, 147.61, 147.20, 146.53, 135.04, $132.21,119.59,116.66,108.13,106.54,106.37,101.03,100.96,87.62,82.00$, $70.88,69.66,54.63,50.12$.

\section{Horsfieldin:}

$\mathrm{mp} 162-165^{\circ} \mathrm{C}\left(\mathrm{Lit}^{7} 164-165^{\circ} \mathrm{C}\right)$.

IR $v_{\max }\left(\mathrm{CCl}_{4}\right) 3550(\mathrm{~b}), 2960,2860,1610,1520,1500,1490,1450,1240,1090$, $1045,970,790,780$.

${ }^{1} \mathrm{H}$ NMR (200 MHz, $\mathrm{CDCl}_{3}$ ) (8ppm) 9.22, s, 1H, OH; 7.00-6.88, m, 6H, ArH; 5.91, $2 \mathrm{H}, \mathrm{s}, \mathrm{OCH}_{2} ; 4.93, \mathrm{~J}=5 \mathrm{~Hz}, 1 \mathrm{H}, \mathrm{ArCHO}, 4.53, \mathrm{~d}, \mathrm{~J}=7 \mathrm{~Hz}, 1 \mathrm{H}, \mathrm{ArCHO} ; 4.16, \mathrm{~d}$, $\mathrm{J}=9 \mathrm{~Hz}, 1 \mathrm{H}, \mathrm{CH} ; 3.93, \mathrm{~s}, 3 \mathrm{H}, \mathrm{OCH}_{3}, 3.63-3.53, \mathrm{~m}, 2 \mathrm{H}, \mathrm{CH}_{2} ; 3.3-3.21, \mathrm{~m}, 2 \mathrm{H}, \mathrm{CH}_{2}$; 
2.87-2.7 $\mathrm{m}, 1 \mathrm{H}, \mathrm{CH}$. Mass spectra $(\mathrm{m} / \mathrm{z})$ (rel.int.\%) 356(56) [M]+, 163(22), 161(26), 152(33), 151(91), 150(51), 149(100), 124(14), 123(23); 122(13), 121(20). Horsfieldin and asarinin were identified by ${ }^{1} \mathrm{H} \mathrm{NMR},{ }^{13} \mathrm{C}$ NMR, MS and mp. ${ }^{7}$

\section{Acknowledgement}

We thank the late Dr.(Mrs.) Rukmini Jayawardena, Dept. of Botany, University of Kelaniya, for identifying the plant, Prof. J.D.Connolly, Dept.of Chemistry, University of Glasgow, Dr. L.K.G.Wickramasinghe, Dept. of Chemistry, University of Kelaniya and the Chemistry Depts. of Universities of Otago (New Zealand) and Arizona(USA) for the spectral data.This research was supported by NARESA grant RG/86/C/1.

\section{References}

1. Willis J.C. (1973). A Dictionary of flowering plants and ferns. (8th ed). pp. 771. University Press, Cambridge.

2. Trimen H. (1974). A handbook of the flora of Ceylon. Vol 3: pp. 433-436. M/S Periodical - Experts, Delhi.

3. Jayaweera D.M.A. (1982). Medicinal plants used in Ceylon. Part 4. pp. 103. National Science Council of Sri Lanka.

4. Kumar N.S., Herath H.M.T.B.\& Karunaratne V. (1988). Aryl alkanone from Myristica dactyloides. Phytochemistry 27(2): 465 - 468.

5. Cooray N.F., Jansz E.R., Wimalasena S., Wijesekara T. P. \& Nair B.M.(1987). Acyl resorcinols from seed kernels of Myristica dactyloides. Phytochemistry 26(12): 3369 - 3371.

6. Purushothaman K.K., Sarada A.\& Connolly J.D. (1977). Malabaricones A-D novel diaryl nonanoids from Myristica malabarica Lam (Myristicaceae). Journal of the Chemical Society. Perkin Trans 1(5): 587 - 588.

7. Gunatilaka A.A.L., De Silva A.M.Y.J., Sotheeswaran S. \& Tillekerathne L.M.V. (1982). Horsfieldin, a lignan and other constituents from Horsfieldia iryaghedhi. Phytochemistry 21(11): 2719-2723.

8. Tillekeratne L.M.V., Jayamanne D.T., Weerasuriya KD.V. \& Gunatilaka A.A.L. (1982). Lignans of Horsfieldia iryaghedhi. Phytochemistry 21(2): 476-478.

9. Packiyasothy E.V., Sarathkumar S.J., Cooray N.F., Weeraratne S. \& Jansz E.R. (1984). Series on Sri Lankan spices. Monograph No.3-Nutmeg \& Mace, p. 8. C.I.S.I.R. Colombo.

10. West R.C. (1974 - 1975). Handbook of chemistry \& physics ( 55 th ed ). C-506. CRC Press, Ohio. 